\title{
Monitoring of Moisture in Transformer Oil Using Optical Fiber as Sensor
}

\author{
S. Laskar and S. Bordoloi \\ Department of EEE, Don Bosco College of Engineering and Technology, Assam Don Bosco University, Guwahati, \\ Assam 781017, India
}

Correspondence should be addressed to S. Laskar; shakuntalalaskar@sify.com

Received 26 March 2013; Revised 21 June 2013; Accepted 1 July 2013

Academic Editor: Luciano Mescia

Copyright (C) 2013 S. Laskar and S. Bordoloi. This is an open access article distributed under the Creative Commons Attribution License, which permits unrestricted use, distribution, and reproduction in any medium, provided the original work is properly cited.

\begin{abstract}
This paper describes an optical fiber sensor and temperature sensor-based instrumentation system to measure the moisture content in transformer oil. The sensor system consists of (i) Diode Laser Source, (ii) a bare and bent multimode fiber as sensor probe, (iii) an LDR as detector, (iv) LM35-based temperature sensor, and (v) microcontroller system having a trained ANN for processing and calibration. The bare and bent optical fiber sensor and the temperature sensor LM35 are used to provide the measures of refractive index (RI) and temperature of a transformer oil sample. An ATmega32-microcontroller-based system with trained ANN algorithm has been developed to determine the moisture content of the transformer oil sample by sampling the readings of the bare bent optical fiber sensor and the temperature sensor.
\end{abstract}

\section{Introduction}

Fiber optic sensors offer unique advantages, such as immunity to electromagnetic interferences, stability, repeatability, durability against harsh environment, and fast response. Therefore, they are used for the measurement of physical parameters such as temperature [1], pressure [2], acceleration [3], curvature measurement [4], hydrocarbon monitoring [5], and host of other applications.

Power transformers are one of the most expensive investments in electric power systems [6]. They are fundamental components of electric power systems, and their reliability is an important factor in the operation of the system [7]. The transformer oil is a good insulating material. However, due to some inevitable factors, some unwanted particles like water and gas can contaminate the oil. As a result, insulation strength gets reduced that may result in partial discharge of transformer oil [8]. Model-based online detection method [6], partial discharge method [7], chromatography, radiofrequency method, infrared spectroscopy, neural network method [9], and so forth are some of the techniques developed for detection of moisture content of transformer oil.
The dielectric parameter $\left(\varepsilon_{r}\right.$, relative permittivity $)$ of transformer oil indicates the quality of the oil as the insulation property is concerned. Again, square of refractive index is represented as relative permittivity of an optical material. Therefore, the moisture content in transformer oil sample can be related to the change in refractive index of the sample. In fact, the measure of refractive index provides information about the turbidity of a liquid [10]. An optical fiber sensor for measuring the refractive index of liquid, based on the measurement of the input and output power of a multimode fiber, has been proposed in $[11,12]$.

Temperature affects the dielectric property of transformer oil [13] and hence the refractive index. Therefore, it is necessary to relate the change in refractive index of transformer oil with its temperature [14].

Microcontroller offers enormous flexibility and opportunity to researchers to investigate and develop sensor-based system according to their requirements and applications. Several microcontrollers-based instrumentation systems were developed using optical fiber as a sensor [1,2]. An electronic microcontroller-based system for high-pressure measurement using a fiber-optic sensor has been proposed [15]. A 


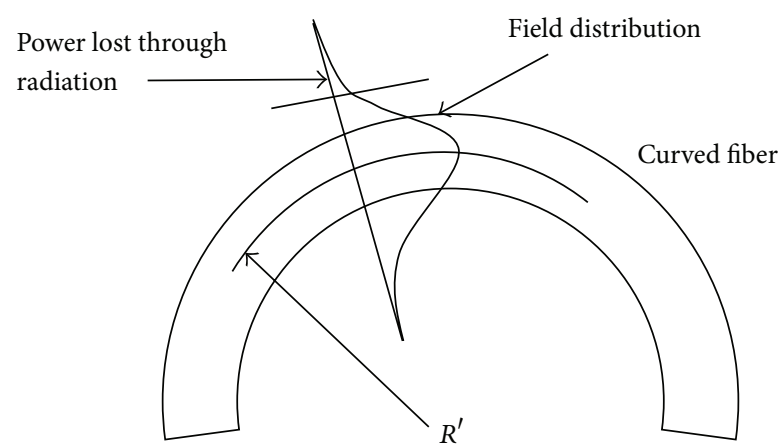

FIgURE 1: Schematic representation of the radiation loss of a mode at a fiber bend.

digital microcontroller-based transducer has been proposed for liquid level measurement, using two optical fibers [16].

This paper describes a microcontroller-based instrumentation system using optical fiber and a temperature IC sensor to measure the moisture content in transformer oil. A bare and bent multimode optical fiber is used as sensor to measure the RI of a transformer oil sample. The optical fiber sensor is prepared by first removing a length of $5 \mathrm{~cm}$ of jacket and cladding of a plastic multimode fiber, and then the bare portion of the fiber is made into a bend of fixed radius of curvature. The bare and bent portion of the multimode optical fiber is termed as "optical sensor probe" (OSP). A laser diode is used to launch a laser beam at one end of the fiber. When the laser beam passes through the bare and bent portion of the optical fiber, intensity modulation of the laser beam takes place, depending upon the RI of a transformer oil sample around it that represents the moisture content of the sample. The laser beam at the other end (output end) of the sensor fiber is detected by an LDR-based potential divider circuit. An ATmega32 microcontroller-based system has been developed to sample analog signals from LDR-based potential divider circuit and from the temperature sensor IC LM35. Since the refractive index of transformer oil depends on the temperature, it is imperative that both refractive index and temperature are to be taken into account to determine the moisture content in a transformer oil sample. For this purpose, a trained artificial neural network is used to process and calibrate the input data. The parameters, such as weightage matrix elements and threshold of the trained ANN, are stored in the microcontroller flash memory to determine moisture content of a transformer oil sample using the input from the potential divider and temperature sensor IC.

\section{Theory of Macrobending}

When a multimode fiber is put at a sharp bend (macrobend) with a radius of curvature exceeding the critical radius of curvature, light rays are lost in the cladding, which results in power loss and thus attenuation. Since higher modes are bound less tightly to the fiber core than the lower order modes, the higher order modes radiate out of the fiber first [17] resulting in loss or attenuation as shown in Figure 1.
For a macrobend multimode fiber, the attenuation coefficient (without any special case of flat or graded profile) is approximately given as [18]

$$
\alpha_{B}=\frac{2 \gamma^{2}(0)}{n_{1} k_{0}} \exp \left[-\frac{2}{3} n_{1} k_{0} R^{\prime}\left(\frac{\beta^{2}-k_{0}^{2} n_{2}^{2}}{n_{1}^{2} k_{0}^{2}}-\frac{2 a}{R^{\prime}}\right)^{3 / 2}\right],
$$

where $R^{\prime}$ represents the radius of curvature, $a$ is the core radius, $n_{1}$ and $n_{2}$ represent the core and cladding RI of the fiber, $\beta$ is the propagation constant, and $k_{0}=\omega / c$.

The propagation constant $\beta$ is expressed in normalized form as

$$
b=\frac{\left(\beta^{2} / k_{0}^{2}-n_{2}^{2}\right)}{n_{1}^{2}-n_{2}^{2}}
$$

where $0 \leq b \leq 1$ for a guided mode

$$
\therefore \beta^{2}-k_{0}^{2} n_{2}^{2}=b k_{0}^{2}\left(n_{1}^{2}-n_{2}^{2}\right) \text {. }
$$

Substituting the expression for $\beta^{2}-k_{0}^{2} n_{2}^{2}$ in (2),

$$
\begin{aligned}
\alpha_{B}= & \frac{2 b k_{0}\left(n_{1}^{2}-n_{2}^{2}\right)}{n_{1}} \\
& \times \exp \left[-\frac{2}{3} n_{1} k_{0} R^{\prime}\left(\frac{b k_{0}^{2}\left(n_{1}^{2}-n_{2}^{2}\right)}{n_{1}^{2} k_{0}^{2}}-\frac{2 a}{R^{\prime}}\right)^{3 / 2}\right],
\end{aligned}
$$

where $n_{2}$ is the RI of the cladding. If all the parameters in (4) are made constant except $n_{2}$, then the attenuation will depend on the cladding material or the surface which will act as a cladding for the fiber.

The loss of optical power (bending, scattering loss, radiation loss, etc.) in an optical fiber is expressed in terms of attenuation coefficient [19] as

$$
\alpha=\frac{10}{l} \log _{10} \frac{P(l)}{P(0)},
$$

where $P(l)$ and $P(0)$ denote the output and input power and $l$ denotes the length of the fiber. The attenuation loss for a short section of the fiber does not scale linearly with length [14]. Rearranging (5),

$$
P(l)=P(0) e^{-0.2304 \alpha l} .
$$

For a multimode fiber with macrobending, the attenuation coefficient in (6) can be expressed as $\alpha=\alpha_{B}$

$$
\therefore P(l)=P(0) e^{-0.2304 \alpha_{B} l} \text {. }
$$

Now expanding the exponential term and neglecting the higher order terms, (7) becomes

$$
P(l)=P(0)\left[1-0.2304 \alpha_{B} l\right] .
$$

If the cladding of the bend portion of the optical fiber is removed and a liquid with refractive index $n_{l}$ is applied 


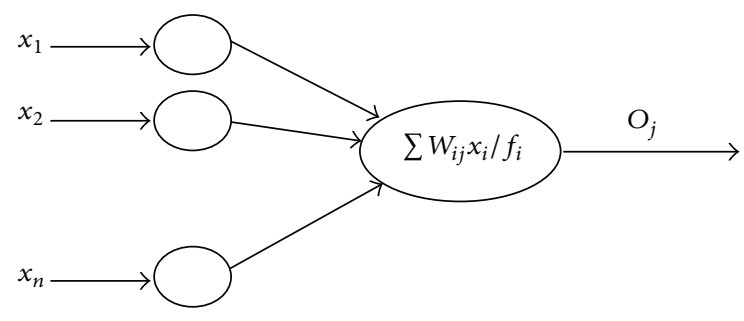

FIgURE 2: An illustration of a processing neuron.

around the bare and bent portion of the optical fiber, (4) and (8) give us

$$
\begin{aligned}
P(l)=P(0)[1 & -\frac{0.4608 b k_{0}\left(n_{1}^{2}-n_{l}^{2}\right) l}{n_{1}} \\
& \left.\times \exp \left\{-\frac{2}{3} n_{1} k_{0} R^{\prime}\left(\frac{b\left(n_{1}^{2}-n_{l}^{2}\right)}{n_{1}^{2}}-\frac{2 a}{R^{\prime}}\right)^{3 / 2}\right\}\right] .
\end{aligned}
$$

Once the bare bent optical fiber sensor is prepared with fixed radius of curvature, $n_{1}, R^{\prime}, k_{0}, b, a$, and $L$ become constant in (9). Again, after fixing the LDR and Diode Laser Source across the optical fiber with proper focusing, $P(0)$, the input power of the laser ray at the input end of the optical fiber also remains constant. Therefore, power associated with the laser beam at the LDR end will depend on the term $\left(n_{1}^{2}-n_{l}^{2}\right)$. Equation (9) shows that the power associated with the laser beam at the LDR end decreases, if the RI $\left(n_{l}\right)$ of a liquid increases due to change in moisture content in it. Thus, the power associated with the laser beam at the LDR end can be represented as function of RI of a liquid, which is applied around the bare bent optical fiber as follows:

$$
P(l)=f\left(n_{1}^{2}-n_{l}^{2}\right) .
$$

The electrical power loss in the LDR of the potential divider circuit shown in Figure 6 can be expressed as

$$
P_{L}=\frac{\left(V_{c c}-V_{x}\right)^{2}}{R_{L}}
$$

where $R_{L}$ is the resistance of the LDR, and it changes with change in the power associated with the laser beam at the LDR end. $V_{x}$ is the analog output signal of the potential divider circuit, which provides the measure of change in $R_{L}$ due to change in RI of the liquid. Thus, electrical power loss in the LDR of the potential divider circuit can be related as the function of (10), as given in the following:

$$
P(l)=f\left(n_{1}^{2}-n_{l}^{2}\right)=f\left(\frac{\left(V_{c c}-V_{x}\right)^{2}}{R_{L}}\right) .
$$

Since $V_{c c}$ and $n_{1}$ are constant, the output of the potential divider circuit $V_{x}$ would provide the measure of RI of a liquid.

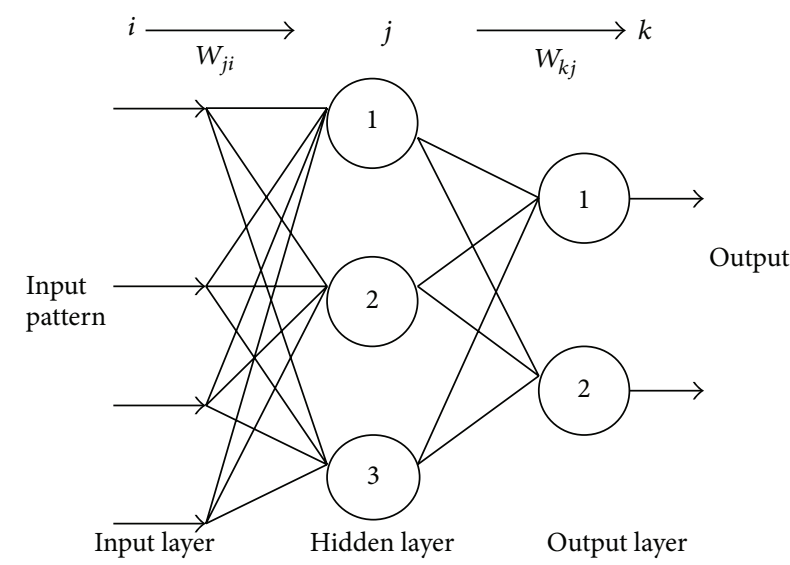

FIGURE 3: Schematic illustration of multilayered feed forward (MLFF) ANN.

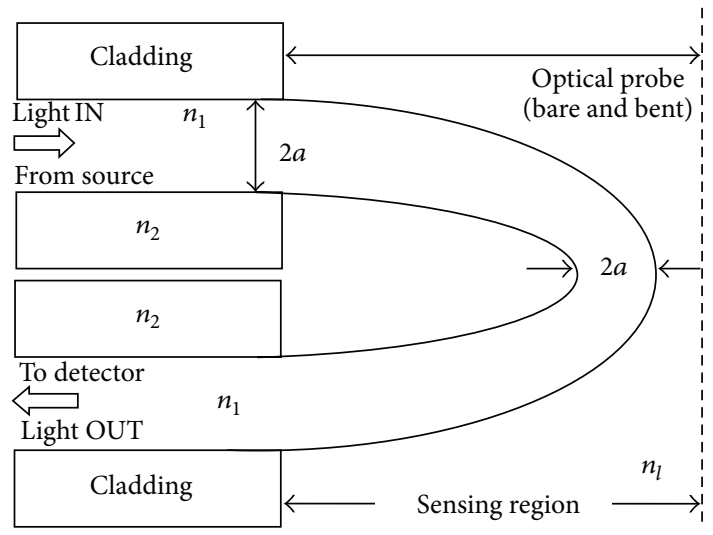

FIGURE 4: Geometry of the proposed sensor.

\section{Artificial Neural Networks (ANN)}

The fundamental unit or building block of a neural network is the neuron [20]. The general neuron has a set of $n$ numbers of inputs $x_{i}$, representing the source of input signals. Each input $x_{i}$ is weighted before reaching the main body of the processing element by the connection strength or the weight factor $W_{j i}$. The input signal is excitatory or inhibitory. In the former case, they increase the activation of the neuron, whilst in the latter they reduce it. The inputs of a particular type are combined together to give the total input to the $j$ th neuron. A schematic illustration of a processing node (PN) is shown in Figure 2.

$\mathrm{O}_{j}$ is the output from the processing node, and the node activation is determined by an output function, which is considered as

$$
f(x)=\frac{1}{1+e^{-x}}
$$

Among the various ANN architectures available in the literatures, the multilayer feed forward (MLFF) network with error back propagation learning algorithm has been selected for this problem mainly because (i) it is the most simple and 


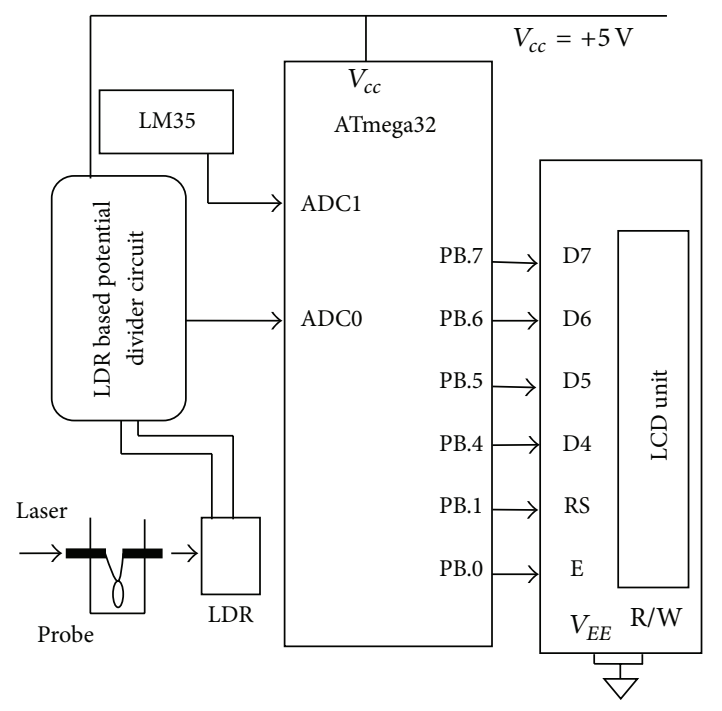

Figure 5: Scheme adopted for the measurement.

comprehensive neural approach for model base and (ii) it has good generalization capability.

\section{Multilayer Feed Forward (MLFF) Network}

In MLFF network the PNs are arranged in layers, and only PNs in adjoint layers are connected [15]. It has a minimum of three layers of PNs: (i) the input layer, (ii) the middle or hidden layer(s), and (iii) the output layer. The information propagation is only in the forward direction, and there are no feedback loops. A MLFF network topology is shown in Figure 3.

The MLFF network uses separate stages for learning and operation. The learning problem is stated as for given a set of input-output pair (cycle/pattern) $\left(x_{1}, O_{1}\right) \cdots\left(x_{n}, O_{n}\right)$, the connected weightage matrix element $W_{j i}$ for each connected ANN is determined in such a way that the network maps $x_{i}$ to $O_{i}$ for $i=1, \ldots, n$, as closely as possible. The error back propagation generalized delta-rule technique is used to train the MLFF network. Back propagation technique and interconnection weightage matrix element $W_{j i}$ are adjusted such that the error function

$$
E=\sum_{p-1}^{P} \sum_{k-1}^{n}\left(d_{k}^{P}-O_{k}^{P}\right)^{2}
$$

is minimized, where

$d_{k}^{P}=$ desired output from $k$ th node in $P$ th training pattern;

$O_{k}^{P}=$ actual output from $k$ th node in $P$ th training pattern;

$P=$ the number of training pattern to input layer;

$n=$ number of nodes in the output layer.

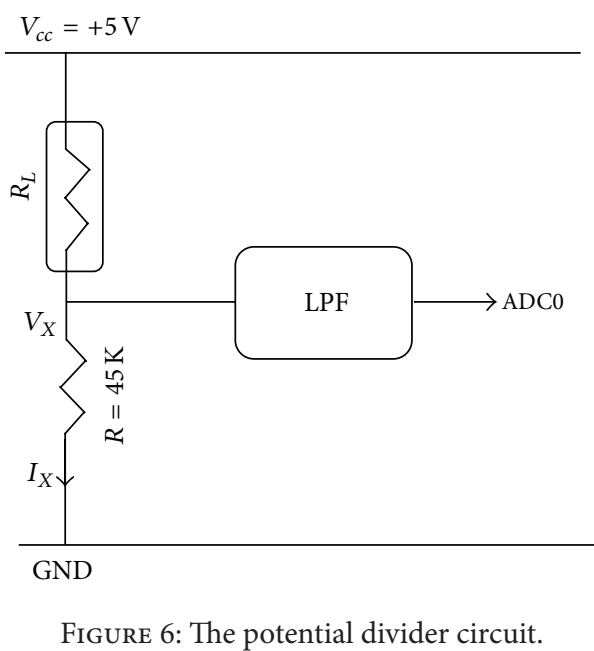

The maximization process is based on gradient descent algorithm. The interconnecting weights between $j$ th layer neurons and $i$ th layer neurons are modified using the following relationship:

$$
W_{j i}^{\mathrm{new}}=W_{j i}^{\mathrm{old}}+\eta \delta_{j} O_{i}+\alpha\left[\Delta W_{j i}^{\mathrm{old}}\right]
$$

If $\mathrm{PN}_{j}$ is an output layer $\mathrm{PN}$, then

$$
\delta_{o k=\left(d_{k}^{P}-\mathrm{O}_{k}^{P}\right)} \frac{\partial f}{\partial x} .
$$

If $\mathrm{PN}_{j}$ is a hidden layer $\mathrm{PN}$, then

$$
\delta_{h j}^{P}=\left(\sum_{k} \delta_{o k}^{P} W_{k j}^{P}\right) \frac{\partial f}{\partial x_{i}},
$$

where $k$ is overall PNs in the layer above the $j$ th layer $\mathrm{PN}$ and $\eta$ and $\alpha$ are the learning rate and momentum factor. The momentum factor and learning rate help in faster convergence of the algorithm. Once network gets trained, the resulting connection weights $W_{j i}$ are stored. In the operation stage, the trained network is used to compute outputs from a set of inputs.

\section{Description of the Fiber Optic Sensor}

The optical fiber sensor used for monitoring the refractive index of transformer oil is a plastic clad silica core multimode fiber. The fiber has a dimension of 200/230 with diameter $500 \mu \mathrm{m}$, the RI of the core of the fiber $\left(n_{1}\right)$ is 1.48 , and cladding $n_{2}$ is 1.46 . The length of the fiber is $60 \mathrm{~cm}$ from which a length of $5 \mathrm{~cm}$ has been unclad by mechanical stripping around the centre. The bare portion of the fiber is then made into a bend of fixed radius of curvature which constitutes the optical sensor probe (OSP). The geometry of the fiber is shown in Figure 4 . 
TABLE 1: Measure of refractive index of transformer oil samples with different moisture content and temperature.

\begin{tabular}{lcccccc}
\hline & Temperature & \multicolumn{5}{c}{ Digital readings of microcontroller system with different percentage of oil-water mixture } \\
In ${ }^{\circ} \mathrm{C}$ & Digital value & $0 \%$ & $0.2 \%$ & $0.8 \%$ & $2 \%$ & $3 \%$ \\
\hline 50 & 102 & 204 & 312 & 356 & 425 & 438 \\
45 & 092 & 141 & 283 & 270 & 368 \\
40 & 081 & 125 & 180 & 194 & 235 \\
35 & 071 & 82 & 128 & 95 & 261 \\
30 & 061 & 60 & & 88 & 137 & 104 \\
\hline
\end{tabular}

\section{Experimental Setup for the Instrumentation System}

Figure 5 shows the configuration of the experimental setup used for the measurement of moisture content of a transformer oil sample. The setup consists of an optical sensor, light source, detector, temperature sensor, and a microcontroller system. A Diode Laser Source (Optochem International, Power $5 \mathrm{~mW}$ ) is used for launching a laser beam into the input end of a multimode optical fiber by immersing the bare bent portion of the fiber into a transfer oil sample. When the laser beam passes through the bare and bent portion of the optical fiber, intensity modulation of the laser beam takes place, depending upon the RI of the transformer oil sample around it that represents the moisture content of the sample. The laser beam at the other end (output end) of the sensor fiber is detected by an LDR-based potential divider circuit. The output from the potential divider circuit via a low pass filter (LPF) is interfaced to an ATmega32 microcontroller ADC (pin number 0, ADC0, analog channel-0). A LM35 temperature sensor is used to read the temperature of the oil samples at the same time. The output of the LM35 sensor is fed to ADC (pin number 1, ADC1, analog channel-1) of microcontroller ATmega32. The display unit of the microcontroller system consists of $16 \times 2$ (i.e., two rows having 16 character LCD display) LCD display units. The LCD has been configured as a $5 \times 7$ dot matrix 4-bit mode character display. It is interfaced to PORT-B of microcontroller ATmega32 for display control.

The potential divider circuit (shown in Figure 6) consists of an LDR in series with a fixed resistance $(45 \mathrm{k} \Omega)$. The output of the circuit is applied to a low pass filter circuit having a cut-off frequency of $8 \mathrm{~Hz}$ to remove the high frequency noises associated with the measurement circuitry.

\section{Experimental Procedures}

The experimental procedure adopted for the generation of data sample to train the ANN requires the following steps.

(i) The bare and bent fiber is cleaned by dipping it in $100 \%(\mathrm{v} / \mathrm{v})$ ethanol and allows it to dry.

(ii) Immersed the bare and bent portion of the optical fiber in a transformer oil sample.

(iii) The microcontroller-based system samples the values of $V_{\text {test liquid }}$ and the temperature. (iv) Repeat steps (i)-(iii) with different transformer oil samples with different moisture content with different temperature.

(v) Using these measurements, an ANN is trained. Software has been developed to implement the algorithm of the trained ANN in the microcontroller-based system. To determine the moisture content of a given transformer oil sample the microcontroller-based system samples the RI and temperature of the sample through $\mathrm{ADC} 0$ and $\mathrm{ADC1}$, and using algorithm of the trained ANN the moisture content of the sample is displayed in the LCD unit.

\section{Results and Discussion}

To generate data cycles to train an ANN for the purpose of correlating the measure of RI and temperature of transformer oil samples to the moisture contents of the samples, standard transformer oil samples with known percentages of water contents are prepared. These transformer oil samples are then subjected to temperature variation from $30^{\circ}$ to $50^{\circ} \mathrm{C}$, and the microcontroller-based system is used to samples the measure of temperature and RI of the transformer oil samplers. The experimental results with different percentages of moisture content and temperatures for different transformer oil samples are presented in Table 1.

The plot for concentration of different transformer oil samples versus the measure of RI of the transformer oil samples with variation of temperature is shown in Figure 7.

The ANN used for the purpose of correlating the measure of RI and temperature of transformer oil samples to their moisture contents contains two neurons at the input layer, four neurons at the hidden layer, and one neuron at the output layer as shown in Figure 8. The measure of temperature and refractive index represented in Table 1 are taken as the input to the ANN input layer. The output layer of the ANN with one neuron provides the moisture content of the transformer oil sample.

The experimental results presented in the Table 1 provided 25 training cycles for temperature range $30^{\circ}-50^{\circ} \mathrm{C}$ and moisture content range from 0.0 to $3.0 \%$. Back propagation technique is used to train the ANN using these 25 data cycles. The training of the ANN is terminated, when difference between calculated output from the ANN and desired output becomes less than 0.0001 . The variation of error at the 


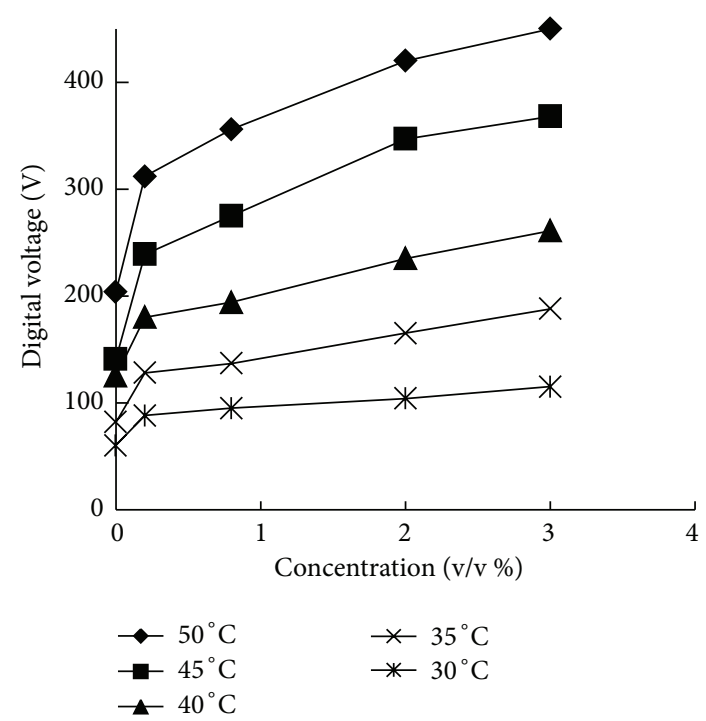

Figure 7: Concentration versus output voltages.

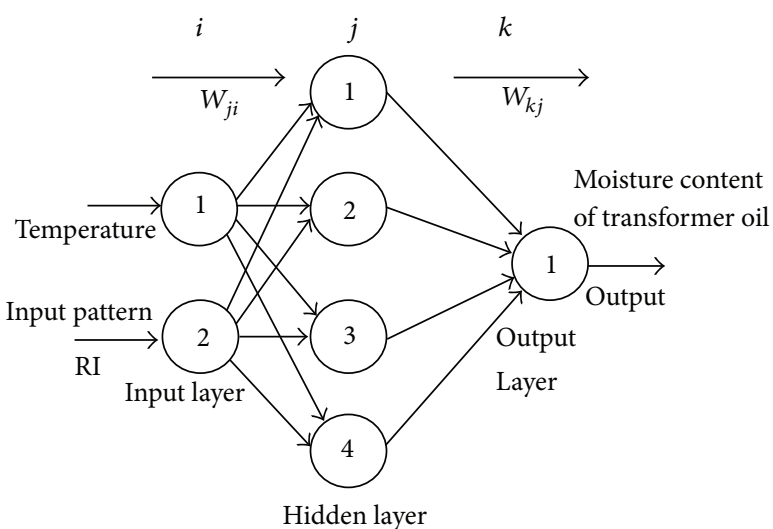

FIgURE 8: Configuration of MLFF ANN used to correlate the measure of RI and temperature of a transformer oil sample to its moisture content.

output neuron with respect to the iteration count is shown in Figure 9.

The weightage matrix elements for the trained ANN are provided in the appendix. The ANN algorithm with these trained weightage matrix elements is implemented in the microcontroller-based system to determine the percentage moisture content of transformer oil by measuring its refractive index and temperature.

It has been observed that, without the LPF, the reading of the LDR-based potential divider circuit keeps fluctuating by -25 to +25 decimal values around the base value. This is due to the noise of the electronic devices and the high frequency noise of the power supply. The use of a passive LPF circuit (R-C circuit) with cut-off frequency of $8 \mathrm{~Hz}$ brings down this fluctuation by -2 to +2 only.

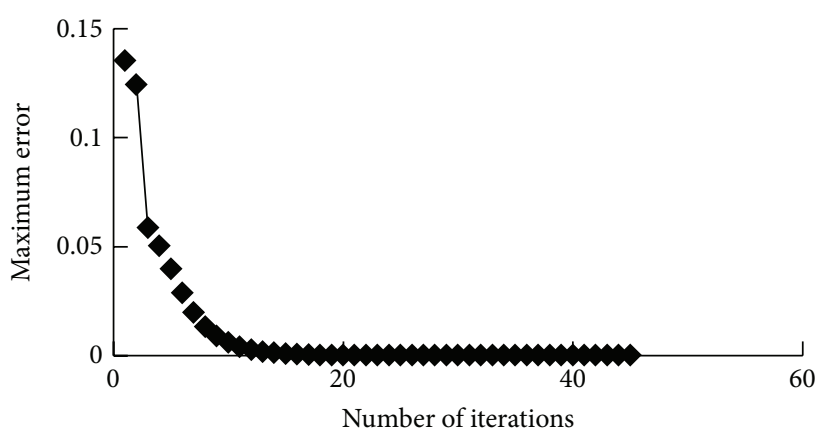

FIGURE 9: Error curve for the MLFF network.

\section{Conclusions}

This paper describes an instrumentation system to measure moisture content in a transformer oil sample using the measure of RI and temperature of the sample. A bare and bent multimode optical sensor is used to measure the RI of the sample, and LM35 is used as temperature sensor. The noise is associated with the measurement (measurement of RI). To generate data cycles to train an ANN for the purpose of correlating the measure of RI and temperature of transformer oil samples to the moisture contents of the samples, standard transformer oil samples with known percentages of water contents are prepared. These transformer oil samples are then subjected to temperature variation from $30^{\circ}$ to $50^{\circ} \mathrm{C}$, and the microcontroller-based system is used to samples the measure of temperature and RI of the transformer oil samples. Software has been developed to implement the algorithm of the trained ANN in the microcontroller-based system. Therefore, the microcontroller-based system can determine the moisture content of a transformer oil sample at any temperature between temperature range $30^{\circ}-50^{\circ} \mathrm{C}$ by sampling the RI and temperature of the sample through $\mathrm{ADC} 0$ and $\mathrm{ADCl}$.

\section{Appendix}

Weightage matrix elements between input and hidden layers and hidden layer and output layer for the trained ANN are as follows:

(1) between first neuron and the neuron of hidden layer

$$
\begin{aligned}
& W_{11}=-51.237881, \\
& W_{12}=63.035744, \\
& W_{13}=0.2, \\
& W_{14}=0.2,
\end{aligned}
$$

(2) between second neuron and the neuron of hidden layer

$$
\begin{aligned}
& W_{21}=-51.237881, \\
& W_{22}=63.035744, \\
& W_{23}=0.2, \\
& W_{24}=0.2,
\end{aligned}
$$


(3) between hidden layer neurons and output layer neurons

$$
\begin{aligned}
& W_{11}=26.923553, \\
& W_{21}=26.923553, \\
& W_{31}=-27.902664, \\
& W_{41}=-27.902664 .
\end{aligned}
$$

\section{References}

[1] D. Hazarika, K. C. Sarma, and P. K. Sarmah, "Microprocessorbased temperature monitoring system using optical fibers," IEEE Sensors Journal, vol. 9, no. 9, pp. 1025-1028, 2009.

[2] D. Hazarika and D. S. Pegu, "Microcontroller based air pressure monitoring instrumentation system using optical fibers as sensor," Optical Fiber Technology, vol. 19, pp. 83-87, 2013.

[3] D. R. Miers, D. Raj, and J. W. Brthold, "Designed and characterization of optical fiber acceleratometers," in Fiber Optic and Laser Sensors V, vol. 838 of Proceedings of SPIE, pp. 314-317, San Diego, Calif, USA, 1987.

[4] R. Liu, Z. Fu, Y. Zhao, Q. Cao, and S. Wang, "Operation principle of a bend enhanced curvature optical fiber sensor," in Proceedings of the IEEE/RSJ International Conference on Intelligent Robots and Systems (IROS '06), pp. 1966-1971, October 2006.

[5] F. Prudenzano, L. Mescia, L. A. Allegretti et al., "Design of an optical sensor array for hydrocarbon monitoring," Optical and Quantum Electronics, vol. 41, no. 1, pp. 55-68, 2009.

[6] W. Hribernik, G. Pascoli, and K. Fróhlich, "An advanced modelbased diagnosis system for online detection of the moisture content of power transformer insulations," in Proceedings of the IEEE International Symposium on Electrical Insulation (ISEI '08), pp. 187-191, June 2008.

[7] G. F. C. Veloso, L. E. B. Silva, I. Noronha, and G. Lambert Torres, "Using partial discharge as sample signal source to identify contamination moisture pattern in power transformer insulating oil," in Proceedings of the 36th Annual Conference of the IEEE Industrial Electronics Society (IECON '10), pp. 10411044, November 2010.

[8] C.-P. Zhu, Y.-L. Huang, M.-L. Shan, and L.-H. Lu, “The research of moisture detection in transformer oil based on ultrasonic method," in Proceedings of the 2nd International Conference on Information Science and Engineering (ICISE '10), pp. 1621-1624, December 2010.

[9] W.-G. Chen, D.-G. Gan, and Q. Liu, "On-line monitoring model based on neural network for moisture content in transformer oil," High Voltage Engineering, vol. 33, no. 5, pp. 73-78, 2007.

[10] A. Banerjee, S. Mukherjee, R. K. Verma et al., "Fiber optic sensing of liquid refractive index," Sensors and Actuators B, vol. 123, no. 1, pp. 594-605, 2007.

[11] T. Takeo and H. Hattori, "Optical fiber sensor for measuring refractive index," Japanese Journal of Applied Physics, vol. 21, no. 10, pp. 1509-1512, 1982.

[12] A. L. Chaudhari and A. D. Shaligram, "Multi-wavelength optical fiber liquid refractometry based on intensity modulation," Sensors and Actuators A, vol. 100, no. 2-3, pp. 160-164, 2002.

[13] R. Shoureshi, V. Permana, R. Wood, R. Swartzendruber, and M. Simoes, "Optical sensor for transformer monitoring," Intelligent Transformer Monitoring System Utilizing Neuro-Fuzzy Technique Approach, PSERC 04-27, 2004.
[14] M. Koch, S. Tenbohlen, and T. Stirl, "Advanced online moisture measurement in power transformer," in Proceedings of the International Conference on Condition Monitoring and Diagnosis (CMD '06), April 2006.

[15] A. Barwicz and W. J. Bock, "An electric high-pressure measuring system using a polarimetric fiber-optic sensor," IEEE Transactions on Instrumentation and Measurement, vol. 39, no. 6, pp. 976-981, 1990.

[16] G. Betta, A. Pietrosanto, and A. Scaglione, "Microcontrollerbased performance enhancement of an optical fiber level transducer," IEEE Transactions on Instrumentation and Measurement, vol. 47, no. 2, pp. 489-493, 1998.

[17] G. Keiser, Attenuation: Optical Fiber Communication, Mcgraw Hill, 2nd edition, 1991.

[18] D. Gloge, "Bending loss in multimode fibers with graded and ungraded core fibers," Applied Optics, vol. 11, no. 11, pp. 25062513, 1972.

[19] B. P. Pal, "Characterization of optical fibers for telecommunication and sensors-Multimode fibers: fundamentals of fibre optics," in Telecommunication and Sensor Systems, pp. 264-267, New Age International, 1st edition, 2009.

[20] D. Hazarika, K. C. Sarma, and N. Baruah, "static voltage stability indication in a power system using ANN," IJMAN, vol. 2, no. 1, pp. 44-49, 2012. 

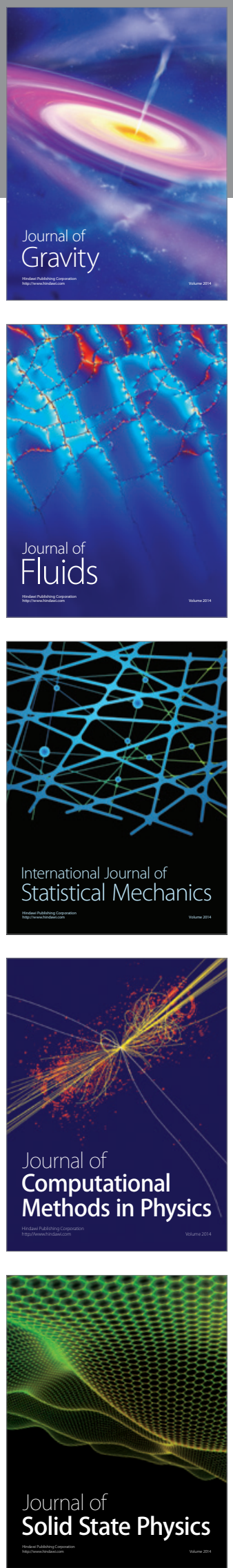

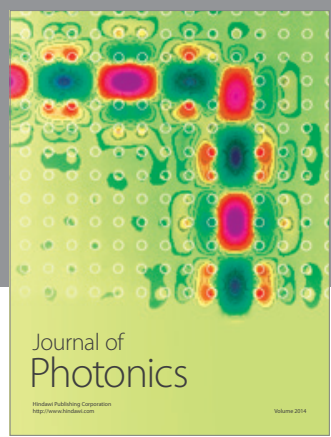

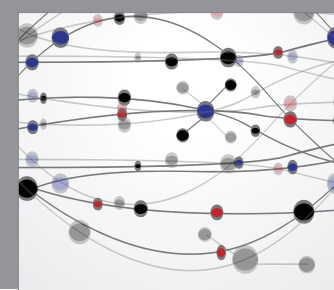

The Scientific World Journal

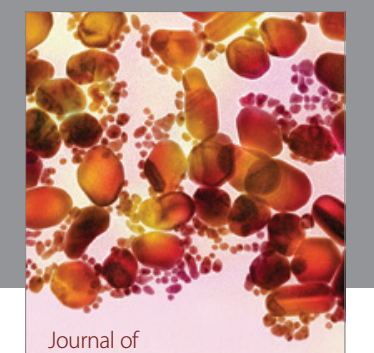

Soft Matter
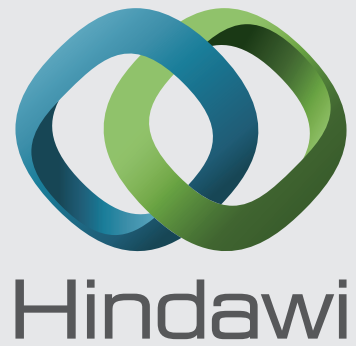

Submit your manuscripts at

http://www.hindawi.com
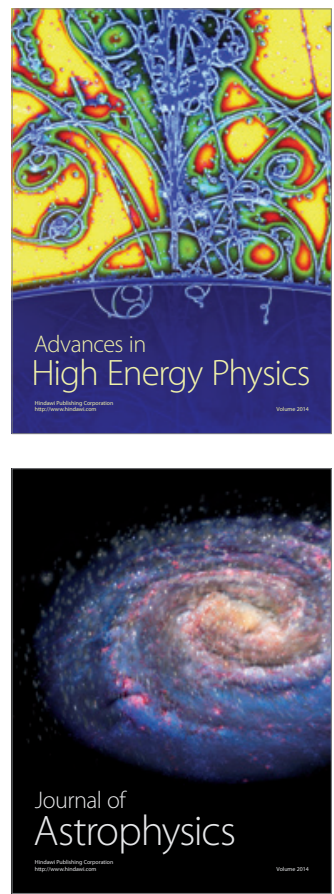
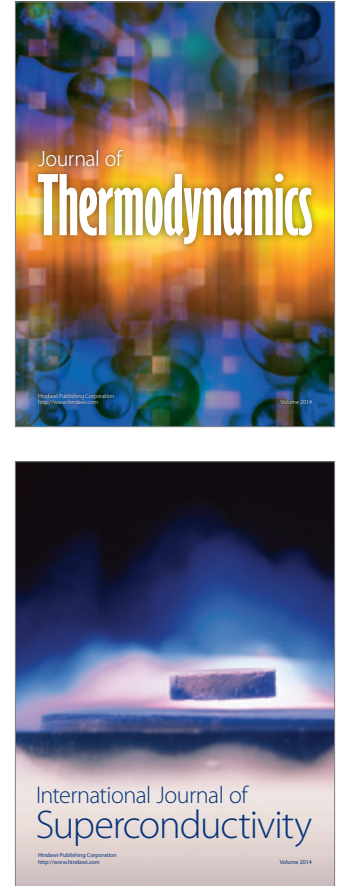
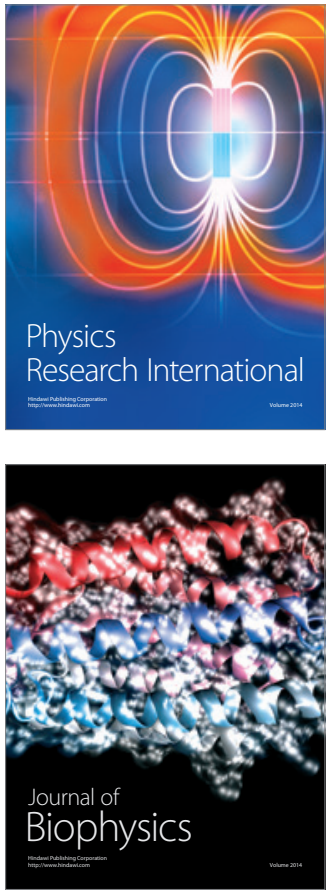
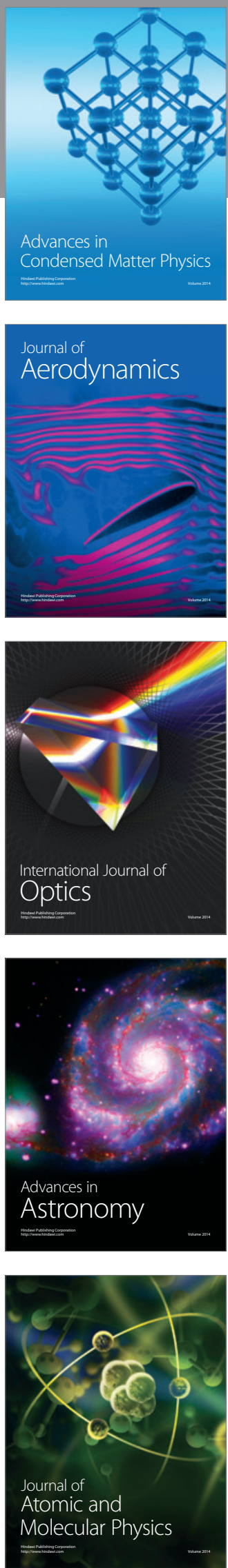